\title{
An Improved Model for the Turbulent PBL
}

Y. Cheng, V.M. Canuto* and A.M. Howard

NASA-Goddard Institute for Space Studies

2880 Broadway, New York, N.Y. 10025

* Dept. of Applied Physics and Mathematics,

Columbia University, New York, N.Y. 10027

Journal of the Atmospheric Sciences, 2000 


\begin{abstract}
Second order turbulence models of the Mellor and Yamada type have been widely used to simulate the PBL. It is however known that these models have several deficiencies. For example, they all predict a critical Richardson number which is about four times smaller than the Large Eddy Simulation (LES) data, they are unable to match the surface data, and they predict a boundary layer height lower than expected.

In the present model, we show that these difficulties are all overcome by a single new physical input: the use of the most complete expression for both the pressure-velocity and the pressure-temperature correlations presently available. Each of the new terms represents a physica' process that was not accounted for by previous models. The new model is presen ied in three different levels according to Mellor and Yamada's terminology: with nery, ready-to-use expressions for the turbulent moments. We show that the new model reproduces several experimental and LES data better than previous models. As far as the PBL is concerned, we show that the model reproduces both the Kansas data as analyzed by Businger et al. in the context of Monin-Obukhov similarity theory for smaller Richardson numb rrs, as well as the LES and laboratory data up to Richardson numbers of order unity. We also show that the model yields a higher PBL height than the previous models.
\end{abstract}




\section{Introduction}

Reynolds stress turbulence modeling began in the early 40's (Chou 1940, 1945) and since then it has been developed by both physicists and engineers, e.g., Rotta (1951), Lumley and Khajeh-Nouri (1974), Launder et al. (1975), Pope (1975), Zeman and Lumley (1979), Speziale (1991), and Shih and Shabbir (1992). The parameterizations of the turbulence closures have been formulated theoretically, verified experimentally (including comparison with the ever more reliable LES data), and applied to various engineering flows. In the geophysical applications, Mellor and Yamada $(1974,1982)$ pioneered the use of turbulence closure models to study the Planetary Boundary Layer (PBL). The Mellor-Yamada (MY) model and its numerous variants have been more successful in the simulation of the PBL than many of the empirical models and have been widely used to describe the atmospheric PBL and the oceanic mixed layer. The MY models are, however, not without deficiencies. Comparison of MY model results with measured data and LES data show consistent discrepancies, and close examination indicates that the weakness of the model comes from three sources: (1) a crude parameterization for the pressure-velocity and the pressure-temperature correlations. (2) the use of a single "master" length scale (all the length scales corresponding to different processes are assumed to be proportional to a master scale), and (3) a downgradient approximation for the third order turbulent moments. These three aspects can be handled as three independent components in the model development and each of them deserves a separate discussion. Along with many other efforts, the present authors tried to address items (2) and (3) elsewhere (Cheng and Canuto 1994; Canuto et al. 1994, 2000). The present paper concentrates on item (1), i.e., how to improve the parameterization for the pressure correlations, thus generalizing the MY models and improving the comparison with both measured and LES data.

Let's look at the deficiency (1) of the MY models and its variants (e.g., Hassid and Galperin 1983) more closely. Firstly, these models predict too low a critical Richardson number (around 0.2), beyond which the turbulence ceases to exist, while both measurements and LES data (e.g., Webster 1964; Wang et al. 1996) indicate that 
the critical value is around unity. Second, when applied to the surface of the neutral boundary layer, none of these models is capable of differentiating between the vertical and lateral components of the turbulent kinetic energy, $\overline{w^{2}}$ and $\overline{v^{2}}$, in fact, they yield identical expressions for the two, while experiments consistently show that the vertical component is much smaller than the lateral one (Table 1 of Mellor and Yamada, 1982; Nieuwstadt 1984, 1985).

As we will show below, these deficiencies are associated with the oversimplification of the parameterizations of the pressure-velocity correlation $\Pi_{i j}$ and pressuretemperature correlation $\Pi_{i}^{\theta}$ which will be corrected by adopting a more complete expression. Both $\Pi_{i j}$ and $\Pi_{i}^{\theta}$ have been shown to contain a slow (return-to-isotropy) part and a rapid part (Launder et al. 1975; Lumley 1978). The rapid parts of both $\Pi_{i j}$ and $\Pi_{i}^{\theta}$ contain velocity terms related to the mean strain-rate tensor $S_{i j}$, and the vorticity tensor $R_{i j}$, as well as buoyancy terms related to the heat fluxes. In addition, the rapid part of $\Pi_{i}^{\theta}$ also contains a term related to the temperature variance $\overline{\theta^{2}}$. By contrast, MY models of $\Pi_{i j}$ include only the slow part and some of the rapid part (the term proportional to $\epsilon S_{i j}$, where $e$ is the turbulent kinetic energy); for $\Pi_{i}^{\theta}$, only the slow part is included. Since each of these missing terms represents a specific physical process, it seems appropriate and necessary to incorporate them in the model formulation, as we do in the present paper.

In Section 1, we introduce the general problem. In Sections 2 and 3, we describe the basic equations and the new turbulence closure. In Section 4, we derive the general model equations for the $3 \mathrm{D}$ case. In Section 5 , we give the detailed and ready-to-use equations for the PBL. The new model is presented in three different "levels" according to MY's terminology. Model constants are determined in Section 6. In Section 7, we compare the new model and the MY model with measured and LES data, where we can see that the new model matches the measured and LES data better than previous models. Conclusions are presented in Section 8. 


\section{Basic equations}

To model a PBL, we need both mean and turbulent variables. They are:

a. First moments

1) Mean velocity, $U_{i}$ :

$$
\frac{D U_{i}}{D t}=-\frac{\partial}{\partial x_{j}} \tau_{i j}-g_{i}-\frac{1}{\rho} \frac{\partial P}{\partial x_{i}}-2 \epsilon_{i j k} \Omega_{j} U_{k}
$$

2) Mean potential temperature, $\Theta$ :

$$
\frac{D \Theta}{D t}=-\frac{\partial}{\partial x_{j}} h_{j}
$$

where

$$
\frac{D}{D t} \equiv \frac{\partial}{\partial t}+U_{j} \frac{\partial}{\partial x_{j}}, \quad \tau_{i j} \equiv \overline{u_{i} u_{j}}, \quad l_{i} \equiv \overline{u_{i} \theta}
$$

Here $u_{i}$ is the $i t h$ component of the turbulent velocity fluctuation, $g_{i}=(0,0, g)$ is the gravitational acceleration, $P$ is the mean pressure, $\rho$ is the mean density, $\Omega_{j}$ is the rotation of the Earth, $\tau_{i j}$ are the Reynolds stresses and $h_{i}$ is the heat flux.

b. Second moments

1) Reynolds stresses, $\tau_{i j}$ :

$$
\begin{gathered}
\frac{D}{D t} \tau_{i j}+D_{i j}=-\left(\tau_{i k} \frac{\partial U_{j}}{\partial x_{k}}+\tau_{j k} \frac{\partial U_{i}}{\partial x_{k}}\right) \\
+\beta_{i} h_{j}+\beta_{j} h_{i}-\Pi_{i j}-\epsilon_{i j}
\end{gathered}
$$

where

$$
\begin{gathered}
\Pi_{i j} \equiv \overline{u_{i} \frac{\partial p}{\partial x_{j}}}+\overline{u_{j} \frac{\partial p}{\partial x_{i}}}-\frac{2}{3} \delta_{i j} \frac{\partial}{\partial x_{k}} \overline{p u_{k}} \\
\epsilon_{i j} \equiv 2 \nu \overline{\frac{\partial u_{i}}{\partial x_{k}} \frac{\partial u_{j}}{\partial x_{k}}}=\frac{2}{3} \delta_{i j} \epsilon, \quad \beta_{i} \equiv \alpha g_{i} \\
D_{i j} \equiv \frac{\partial}{\partial x_{k}}\left(\overline{u_{i} u_{j} u_{k}}+\frac{2}{3} \delta_{i j} \overline{p u_{k}}\right)
\end{gathered}
$$

Here, $\alpha$ is the volume expansion coefficient, $\Pi_{i j}$ is the pressure-velocity correlation tensor, $\nu$ is the molecular viscosity and $\epsilon$ is the dissipation rate of the turbulent kinetic energy $\epsilon$, and $D_{i j}$ is the diffusion term.

Of special interest is the equation for the turbulent kinetic energy $\epsilon$ :

$$
e=\frac{1}{2} q^{2}, \quad q^{2} \equiv \overline{u_{i} u_{i}}
$$




$$
\frac{D e}{D t}+\frac{1}{2} D_{i i}=-\tau_{i j} \frac{\partial U_{i}}{\partial x_{j}}+\beta_{i} h_{i}-\epsilon
$$

2) Heat flux, $h_{i}$ :

$$
\frac{D}{D t} h_{i}+D_{i}^{h}=-h_{j} \frac{\partial U_{i}}{\partial x_{j}}-\tau_{i j} \frac{\partial \Theta}{\partial x_{j}}+\beta_{i} \overline{\theta^{2}}-\Pi_{i}^{\theta}
$$

where

$$
\Pi_{i}^{\theta} \equiv \overline{\theta \frac{\partial p}{\partial x_{i}}}, \quad D_{i}^{h}=\frac{\partial}{\partial x_{j}} \overline{u_{i} u_{j} \theta}
$$

where $\Pi_{i}^{\theta}$ is the pressure-temperature correlation, and $D_{i}^{h}$ is the diffusion of the heat flux $h_{i}$.

3) Temperature variance, $\overline{\theta^{2}}$ :

$$
\frac{D}{D t} \overline{\theta^{2}}+D_{\theta}=-2 h_{i} \frac{\partial \Theta}{\partial x_{i}}-2 \epsilon_{\theta}
$$

where

$$
\epsilon_{\theta} \equiv \overline{\left(\frac{\partial \theta}{\partial x_{j}}\right)^{2}}, \quad D_{\theta}=\frac{\partial}{\partial x_{i}} \overline{u_{i} \theta^{2}}
$$

where $\chi$ is the molecular conductivity and $D_{\theta}$ is the diffusion of the temperature variance.

In the present study, terms containing the molecular viscosity $\nu$ and molecular conductivity $\chi$ have been neglected, except for $\epsilon_{i j}$ and $\epsilon_{\theta}$. In addition, in the second moment equations, rotation has also been neglected. The modeling of the third-order moments exceeds the scope of the present paper, but the interested readers may refer to recent work on the subject (Canuto et al. 1994, 2000). As already stated, in this paper we concentrate on the closure parameterization of the correlations $\Pi_{i j}$ and $\Pi_{i}^{\theta}$, which will be shown to improve the PBL model results.

\section{Turbulence closure}

\section{a. Pressure correlations}

The pressure correlation terms $\Pi_{i j}$ and $\Pi_{i}^{\theta}$ in Eqs. $(2 \mathrm{~b})$ and $(3 \mathrm{~b})$ contain three distinct contributions due to (1) turbulence self-interactions (the return-to-isotropy or slow part), (2) mean shear-turbulence interactions (a rapid part), and (3) buoyancyturbulence interactions (also a rapid part). The most complete model: for $\Pi_{i j}$ and $\Pi_{i}^{\theta}$ 
are given by (Launder et al. 1975; Zeman and Lumley 1979):

$$
\Pi_{i j}=\Pi_{i j}^{(1)}+\Pi_{i j}^{(2)}+\Pi_{i j}^{(3)}, \quad \Pi_{i}^{\theta}=\Pi_{i}^{\theta(1)}+\Pi_{i}^{\theta(2)}+\Pi_{i}^{\theta(3)}
$$

where

$$
\begin{gathered}
\Pi_{i j}^{(1)}=2 \tau_{p v}^{-1} b_{i j} \\
\Pi_{i j}^{(2)}=-\frac{4}{5} \epsilon S_{i j}-\alpha_{1} \Sigma_{i j}-\alpha_{2} Z_{i j} \\
\Pi_{i j}^{(3)}=\left(1-\beta_{5}\right) B_{i j} \\
\Pi_{i}^{\theta(1)}=\tau_{p \theta}^{-1} h_{i} \\
\Pi_{i}^{\theta(2)}=-\frac{3}{4} \alpha_{3}\left(S_{i j}+\frac{5}{3} R_{i j}\right) h_{j} \\
\Pi_{i}^{\theta(3)}=\gamma_{1} \beta_{i} \overline{\theta^{2}}
\end{gathered}
$$

where $b_{i j}$ is the traceless Reynolds stress tensor defined as follows

$$
b_{i j}=\overline{u_{i} u_{j}}-\frac{2 \epsilon}{3} \delta_{i j}
$$

The other tensors are defined as follows:

$$
\begin{gathered}
S_{i j}=\frac{1}{2}\left(\frac{\partial U_{i}}{\partial x_{j}}+\frac{\partial U_{j}}{\partial x_{i}}\right) \quad R_{i j}=\frac{1}{2}\left(\frac{\partial U_{i}}{\partial x_{j}}-\frac{\partial U_{j}}{\partial x_{i}}\right) \\
\Sigma_{i j}=b_{i k} S_{k j}+S_{i k} b_{k j}-\frac{2}{3} \delta_{i j} b_{k m} S_{m k}, \quad Z_{i j}=R_{i k} b_{k j}-b_{i k} R_{k j} \\
B_{i j}=\beta_{i} h_{j}+\beta_{j} h_{i}-\frac{2}{3} \delta_{i j} \beta_{k} h_{k}
\end{gathered}
$$

where $S_{i j}$ and $R_{i j}$ are shear and vorticity respectively. In most past second-order turbulence models for the PBL, these pressure correlations terms were parameterized much less completely. For example, the MY model and its variants (Mellor 1973, Mellor and Yamada 1974, 1982 and Hassid and Galperin 1983) only consider:

$$
\begin{gathered}
\Pi_{i j}^{(1)}=2 \tau_{p v}^{-1} b_{i j}, \quad \Pi_{i j}^{(2)}=-\frac{4}{5} \epsilon S_{i j}, \quad \Pi_{i j}^{(3)}=0 \\
\Pi_{i}^{\theta(1)}=\tau_{p \theta}^{-1} h_{i}, \quad \Pi_{i}^{\theta(2)}=\Pi_{i}^{\theta(3)}=0
\end{gathered}
$$

In other words, only the slow terms and one single rapid term (the first term in the expression of $\left.\Pi_{i j}^{(2)}\right)$ are retained; most of the rapid terms are neglected, and no buoyancy 
effects on the pressure correlation are included. In Section 6, we will show how these missing terms lead to some of the model deficiencies, e.g., failure to match the data in the neutral surface layer as well as in the stably stratified flows.

\section{Algebraic Reynolds stress and heat flux models (AM)}

a. Prognostic equations

The mean wind $U_{i}$ and mean potential temperature $\Theta$ are solved prognostically using Eqs.(1a-c).

b. A hierarchy of turbulence models

We present our new AM model as a hierarchy of different levels (levels 3, 2.5 and 2). In the level 3 model, the turbulent kinetic energy $\epsilon$ and the temperature variance $\overline{\theta^{2}}$ are solved from their prognostic equations (see Appendix), while the other second moments are solved from algebraic equations. In the level 2.5 model, the prognostic mode] equation for $\overline{\theta^{2}}$ is reduced to an algebraic relation. In the level 2 model, the prognostic equations for both $\epsilon$ and $\overline{\theta^{2}}$ are replaced by corresponding algebraic equations.

The turbulent kinetic energy dissipation rate $\epsilon$ and temperature variance dissipation rate $\epsilon_{\theta}$ could be solved from their prognostic equations, or they could be parameterized. Here we express them in terms of the corresponding dissipation time scales, $\tau$ and $\tau_{\theta}$ :

$$
\epsilon=\frac{2 \epsilon}{\tau}, \quad \epsilon_{\theta}=\frac{\overline{\theta^{2}}}{\tau_{\theta}}
$$

In Section 6 we will discuss a parameterization of $\tau$ and $\tau_{\theta}$.

In the main text of this paper we will concentrate on the level 2.5 and 2 models.

c. Algebraic equations for the second moments

Combining (2a) and (2f), we can obtain the equation for $b_{i j}$, Eq.(5d):

$$
\frac{D}{D t} b_{i j}+D_{i j}=-\frac{4}{3} e S_{i j}-\Sigma_{i j}-Z_{i j}+B_{i j}-\Pi_{i j}
$$


where

$$
D_{i j} \equiv \frac{\partial}{\partial x_{k}} \overline{\left(u_{i} u_{j}-\frac{1}{3} u_{\ell} u_{\ell} \delta_{i j}\right) u_{k}}
$$

Assuming that the left side of ( $8 \mathrm{a}$ ) can be neglected and employing (5b) for the pressure-velocity correlation $\Pi_{i j}$, one obtains the following algebraic equation for $b_{i j}$

$$
b_{i j}=-\lambda_{1} e \tau S_{i j}-\lambda_{2} \tau \Sigma_{i j}-\lambda_{3} \tau Z_{i j}+\lambda_{4} \tau B_{i j}
$$

where

$$
\begin{array}{ccc}
\lambda=\frac{\tau_{p v}}{\tau}, & \lambda_{1}=\frac{4}{15} \lambda, \quad \lambda_{2}=\frac{1}{2}\left(1-\alpha_{1}\right) \lambda \\
\lambda_{3}=\frac{1}{2}\left(1-\alpha_{2}\right) \lambda, & \lambda_{4}=\frac{1}{2} \beta_{5} \lambda
\end{array}
$$

These model constants will be given in Section 6. Similarly, in the prognostic equation (3a) for the heat flux $h_{i}$, if the left side is neglected and (5c) is employed for the pressure-temperature correlation $\Pi_{i}^{\theta}$, one obtains the algebraic equation for $h_{i}$ at level 3 :

$$
A_{i j} h_{j}=-\tau\left(b_{i j}+\frac{2 \epsilon}{3} \delta_{i j}\right) \frac{\partial \Theta}{\partial x_{j}}+\lambda_{0} \tau \beta_{i} \overline{\theta^{2}}
$$

where

$$
\begin{gathered}
A_{i j}=\lambda_{5} \delta_{i j}+\lambda_{6} \tau S_{i j}+\lambda_{7} \tau R_{i j} \\
\lambda_{0}=1-\gamma_{1}, \quad \lambda_{5}=\frac{\tau}{\tau_{p \theta}}, \quad \lambda_{6}=1-\frac{3}{4} \alpha_{3}, \quad \lambda_{7}=1-\frac{5}{4} \alpha_{3}
\end{gathered}
$$

At levels 2.5 and 2, we further simplify the problem by neglecting the left side in the prognostic equation for $\overline{\theta^{2}}$, Eq.(4a), to obtain the algebraic equation

$$
\overline{\theta^{2}}=-\tau_{\theta} h_{i} \frac{\partial \Theta}{\partial x_{i}}
$$

Substituting (11) into (10a), we obtain the algebraic equation for $h_{i}$ at levels 2.5-2:

$$
A_{i j}^{\prime} h_{j}=-\tau\left(b_{i j}+\frac{2 \epsilon}{3} \delta_{i j}\right) \frac{\partial \Theta}{\partial x_{j}}
$$

where

$$
A_{i j}^{\prime}=\lambda_{5} \delta_{i j}+\lambda_{6} \tau S_{i j}+\lambda_{7} \tau R_{i j}+\lambda_{8} \tau^{2} \beta_{i} \frac{\partial \Theta}{\partial x_{j}}
$$

and where

$$
\lambda_{8}=\left(1-\gamma_{1}\right) \frac{\tau_{\theta}}{\tau}
$$




\section{PBL Model}

a. Mean wind and potential temperature equations

In the PBL, several approximations can be made to the equations for the mean wind and temperature. In Eq.(1a), the horizontal pressure gradient can be expressed in terms of the mean geostrophic wind components $U_{g}$ and $V_{g}$ as follows:

$$
\frac{1}{\rho}\left(\frac{\partial P}{\partial x}, \frac{\partial P}{\partial y}\right)=f_{c}\left(V_{s},-U_{g}\right)
$$

and the rotation term can be approximated as

$$
-2 \epsilon_{i j k} \Omega_{j} U_{k}=f_{c} \epsilon_{i j 3} U_{j}
$$

where $x, y$ and $z$ are the eastward, northward and vertical directions respectively, $f_{c}=2 \Omega \sin \phi$ is the Coriolis parameter with $\Omega$ the angular velocity of the Earth and $\phi$ the latitude. In Eq.(1b), the horizontal temperature gradient can be approximated with the thermal wind relation,

$$
\left(\frac{\partial \Theta}{\partial x}, \frac{\partial \Theta}{\partial y}\right)=\frac{f_{c}}{g \alpha}\left(\frac{\partial V_{g}}{\partial z},-\frac{\partial U_{g}}{\partial z}\right)
$$

The equations for the eastward and northward horizontal mean wind components $U$ and $V$ and for the mean potential temperature $\Theta$ in the PBL can then be written as:

$$
\begin{gathered}
\frac{\partial U}{\partial t}=f_{c}\left(V-V_{g}\right)-\frac{\partial \overline{u w}}{\partial z} \\
\frac{\partial V}{\partial t}=-f_{c}\left(U-U_{g}\right)-\frac{\partial \overline{v w}}{\partial z} \\
\frac{\partial \Theta}{\partial t}=-\frac{\partial}{\partial z} \overline{w \theta}+\frac{f_{c}}{g \alpha}\left(V \frac{\partial U_{g}}{\partial z}-U \frac{\partial V_{g}}{\partial z}\right)-W \frac{\partial \Theta}{\partial z}
\end{gathered}
$$

\section{b. Level 3 model}

Since the level 2.5 and level 2 models catch the main features of the second-order closure models and are easy to use, they have become the most popular second-order closure models in the PBL community. We will concentrate on them in the sections below. Yet, the level 3 model has its own strength in that it produces counter gradient 
heat fluxes, a phenomenon observed in the upper part of the PBL. In the Appendix we will present the details of the level 3 model for completeness and for future reference.

\section{c. Level 2.5 model}

In the level 2.5 model, the turbulent kinetic energy $e$ is solved form its prognostic equation:

$$
\frac{\partial e}{\partial t}=-\frac{\partial}{\partial z} \frac{1}{2} \overline{u^{2} w+v^{2} w+w^{3}}-\frac{\partial U}{\partial z} \overline{u w}-\frac{\partial V}{\partial z} \overline{v w}+g \alpha \overline{w \theta}-\epsilon
$$

The differential equation for the temperature variance $\overline{\theta^{2}}$ is given by (11) with the index $\mathrm{i}$ replaced by 3 . From the algebraic equations for $\overline{u_{i} u_{j}}$ and $\overline{u_{i} \theta}$, Eqs.(9a) and (12a), we obtain:

$$
\begin{gathered}
\overline{u^{2}}=\frac{1}{3} q^{2}-\frac{\tau}{3}\left[\left(\lambda_{2}+3 \lambda_{3}\right) \frac{\partial U}{\partial z} \overline{u w}-2 \lambda_{2} \frac{\partial V}{\partial z} \overline{v w}+2 \lambda_{4} g \alpha \overline{w \theta}\right] \\
\overline{v^{2}}=\frac{1}{3} q^{2}-\frac{\tau}{3}\left[\left(\lambda_{2}+3 \lambda_{3}\right) \frac{\partial V}{\partial z} \overline{v w}-2 \lambda_{2} \frac{\partial U}{\partial z} \overline{u w}+2 \lambda_{4} g \alpha \overline{w \theta}\right] \\
\overline{w^{2}}=\frac{1}{3} q^{2}+\frac{\tau}{3}\left[\left(3 \lambda_{3}-\lambda_{2}\right)\left(\frac{\partial U}{\partial z} \overline{u w}+\frac{\partial V}{\partial z} \overline{v w}\right)+4 \lambda_{4} g \alpha \overline{w \theta}\right] \\
\overline{u v}=-\left(\lambda_{2}+\lambda_{3}\right) \frac{\tau}{2}\left(\frac{\partial V}{\partial z} \overline{u w}+\frac{\partial U}{\partial z} \overline{v w}\right) \\
\overline{u w}=-\frac{\tau}{2} \frac{\partial U}{\partial z}\left[\frac{1}{2}\left(\lambda_{1}-\frac{4}{3} \lambda_{2}\right) q^{2}+\left(\lambda_{2}-\lambda_{3}\right) \overline{u^{2}}+\left(\lambda_{2}+\lambda_{3}\right) \overline{w^{2}}\right] \\
-\left(\lambda_{2}-\lambda_{3}\right) \frac{\tau}{2} \frac{\partial V}{\partial z} \overline{u v}+\lambda_{4} \tau g \alpha \overline{u \theta} \\
\overline{v w}=-\frac{\tau}{2} \frac{\partial V}{\partial z}\left[\frac{1}{2}\left(\lambda_{1}-\frac{4}{3} \lambda_{2}\right) q^{2}+\left(\lambda_{2}-\lambda_{3}\right) \overline{v^{2}}+\left(\lambda_{2}+\lambda_{3}\right) \overline{w^{2}}\right] \\
-\left(\lambda_{2}-\lambda_{3}\right) \frac{\tau}{2} \frac{\partial U}{\partial z} \overline{u v}+\lambda_{4} \tau g \alpha \overline{v \theta} \\
\overline{u \theta}=-\lambda_{5}^{-1} \tau\left[\frac{\partial \Theta}{\partial z} \overline{u w}+\frac{1}{2}\left(\lambda_{6}+\lambda_{7}\right) \frac{\partial U}{\partial z} \overline{w \theta}\right]
\end{gathered}
$$




$$
\begin{gathered}
\overline{v \theta}=-\lambda_{5}^{-1} \tau\left[\frac{\partial \Theta}{\partial z} \overline{v w}+\frac{1}{2}\left(\lambda_{6}+\lambda_{7}\right) \frac{\partial V}{\partial z} \overline{w \theta}\right] \\
\overline{w \theta}=-\lambda_{5}^{-1} \tau\left[\frac{\partial \Theta}{\partial z} \overline{w^{2}}+\frac{1}{2}\left(\lambda_{6}-\lambda_{7}\right)\left(\frac{\partial U}{\partial z} \overline{u \theta}+\frac{\partial V}{\partial z} \overline{v \theta}\right)\right]\left[1+\lambda_{5}^{-1} \lambda_{8} g \alpha \tau^{2} \frac{\partial \Theta}{\partial z}\right]^{-1}
\end{gathered}
$$

Eqs.(15) can be solved using symbolic algebra. The results are:

$$
\begin{gathered}
(\overline{u w}, \overline{v w})=-K_{M}\left(\frac{\partial U}{\partial z}, \frac{\partial V}{\partial z}\right) \\
\overline{w \theta}=-K_{H} \frac{\partial \Theta}{\partial z} \\
K_{M}=e \tau S_{M}, \quad K_{H}=e \tau S_{H} \\
S_{M}=\frac{1}{D}\left(s_{0}+s_{1} G_{H}+s_{2} G_{M}\right) \\
S_{H}=\frac{1}{D}\left(s_{4}+s_{5} G_{H}+s_{6} G_{M}\right)
\end{gathered}
$$

where $G_{H}$ and $G_{M}$ are the dimensionless gradients for the mean potential temperature and the mean velocity

$$
\begin{gathered}
G_{H} \equiv(\tau N)^{2}, \quad G_{M} \equiv(\tau S)^{2} \\
N^{2} \equiv g \alpha \frac{\partial \Theta}{\partial z}, \quad S^{2} \equiv\left(\frac{\partial U}{\partial z}\right)^{2}+\left(\frac{\partial V}{\partial z}\right)^{2}
\end{gathered}
$$

and

$$
\begin{gathered}
D=d_{0}+d_{1} G_{H}+d_{2} G_{M}+d_{3} G_{H}^{2}+d_{4} G_{H} G_{M}+d_{5} G_{M}^{2} \\
d_{0}=3 \lambda_{5}^{2}, \quad d_{1}=\lambda_{5}\left(7 \lambda_{4}+3 \lambda_{8}\right) \\
d_{2}=\lambda_{5}^{2}\left(3 \lambda_{3}^{2}-\lambda_{2}^{2}\right)-\frac{3}{4}\left(\lambda_{6}^{2}-\lambda_{7}^{2}\right), \quad d_{3}=\lambda_{4}\left(4 \lambda_{4}+3 \lambda_{8}\right) \\
d_{4}=\lambda_{4}\left[\lambda_{2} \lambda_{6}-3 \lambda_{3} \lambda_{7}-\lambda_{5}\left(\lambda_{2}^{2}-\lambda_{3}^{2}\right)\right]+\lambda_{5} \lambda_{8}\left(3 \lambda_{3}^{2}-\lambda_{2}^{2}\right) \\
d_{5}=\frac{1}{4}\left(\lambda_{2}^{2}-3 \lambda_{3}^{2}\right)\left(\lambda_{6}^{2}-\lambda_{7}^{2}\right), \quad s_{0}=\frac{3}{2} \lambda_{1} \lambda_{5}^{2} \\
s_{1}=-\lambda_{4}\left(\lambda_{6}+\lambda_{7}\right)+2 \lambda_{4} \lambda_{5}\left(\lambda_{1}-\frac{1}{3} \lambda_{2}-\lambda_{3}\right)+\frac{3}{2} \lambda_{1} \lambda_{5} \lambda_{8} \\
s_{2}=-\frac{3}{8} \lambda_{1}\left(\lambda_{6}^{2}-\lambda_{7}^{2}\right), \quad s_{4}=2 \lambda_{5}, \quad s_{5}=2 \lambda_{4} \\
s_{6}=\frac{2}{3} \lambda_{5}\left(3 \lambda_{3}^{2}-\lambda_{2}^{2}\right)-\frac{1}{2} \lambda_{1} \lambda_{5}\left(3 \lambda_{3}-\lambda_{2}\right)+\frac{3}{4} \lambda_{1}\left(\lambda_{6}-\lambda_{7}\right)
\end{gathered}
$$




\section{d. Realizability conditions for level 2.5 model}

Realizability conditions are common to all second-order closure models. For the present 2.5 level model, there are a few limitations on the values of $S_{M}$ and $S_{H},(17 \mathrm{a})$ and (17b). Specifically, the two variables $G_{M}$ and $G_{H}$ must be limited to certain domains outside of which the model may produce unphysical results since some underlying assumptions (e.g., that departure from isotropy be small) may no longer be valid.

Let us first consider the limitation on buoyancy. $G_{H}$ may be negative (unstable), zero (neutral) or positive (stable). Assuming that production equals dissipation for the turbulence kinetic energy $e$ [see Eq. (22) below], and taking the limit $G_{M} \rightarrow 0$ and noticing that $G_{M}$ is always non-negative, we have

$$
S_{H}\left(0, G_{H}\right) G_{H}+2>0
$$

Substituting Eq.(17b) into Eq.(20a) yields the relation

$$
G_{H}>\frac{-\left(s_{4}+2 d_{1}\right)+\left[\left(s_{4}+2 d_{1}\right)^{2}-8 d_{0}\left(s_{5}+2 d_{3}\right)\right]^{1 / 2}}{2\left(s_{5}+2 d_{3}\right)}
$$

For the model constants used here (see Section 6), this minimum value of $G_{H}$ is -10.8 ; the negative value indicates that it occurs in the unstable region.

Next, we examine the limitation on the shear number. $G_{M}$ should always be nonnegative. Following Hassid and Galperin (1983), who argue that an increase of shear should not result in a decrease of normalized momentum flux, we apply the following condition,

$$
\frac{d}{d G_{M}}\left[\frac{\left(\overline{u w}^{2}+\overline{v w}^{2}\right)^{1 / 2}}{e}\right] \geq 0
$$

Using Eqs.(16-18), Eq.(21a) can be reduced to a cubic inequality in $G_{M}$,

$$
\begin{aligned}
& s_{2} d_{5} G_{M}^{3}+\left[\left(3 s_{1} d_{5}-s_{2} d_{4}\right) G_{H}+3 s_{0} d_{5}-s_{2} d_{2}\right] G_{M}^{2}+\left[\left(s_{1} d_{4}-3 s_{2} d_{3}\right) G_{H}^{2}\right. \\
& \left.+\left(s_{1} d_{2}+s_{0} d_{4}-3 s_{2} d_{1}\right) G_{H}-3 s_{2} d_{0}+s_{0} d_{2}\right] G_{M}-\left(s_{0}+s_{1} G_{H}\right)\left(d_{3} G_{H}^{2}+d_{1} G_{H}+d_{0}\right)<0
\end{aligned}
$$


Although Eq.(21b) can be solved exactly, one may use the following approximate expression based on the fact that the terms containing $s_{2}$ and $d_{5}$ are relatively small,

$$
G_{M}<\frac{d_{0}+d_{1} G_{H}+d_{3} G_{H}^{2}}{d_{2}+d_{4} G_{H}}
$$

e. Level 2 model

If we assume that production and dissipation equal each other, the differential equation for $\epsilon$, Eq.(14), reduces to

$$
S_{M}\left(G_{M}, G_{H}\right) G_{M}-S_{H}\left(G_{M}, G_{H}\right) G_{H}-2=0
$$

which can be re-written as an equation for $G_{M}$ (or for $G_{H}$ ) that depends on only one parameter, the gradient Richardson nümber,

$$
R i=\frac{G_{H}}{G_{M}}=\frac{N^{2}}{S^{2}}
$$

The resulting equation is:

$$
\left(c_{1} R i^{2}+c_{2} R i+c_{3}\right) G_{M}^{2}+\left(c_{4} R i+c_{5}\right) G_{M}+c_{6}=0
$$

where

$$
\begin{gathered}
c_{1}=s_{5}+2 d_{3}, \quad c_{2}=-s_{1}+s_{6}+2 d_{4}, \quad c_{3}=-s_{2}+2 d_{5} \\
c_{4}=s_{4}+2 d_{1}, \quad c_{5}=-s_{0}+2 d_{2}, \quad c_{6}=2 d_{0}
\end{gathered}
$$

Eq. (23b) is a simple algebraic expression for the variable $G_{M}$. Substituting the $G_{M}$ solved from (23b) into (17a-b) we can plot the stability functions $S_{M}$ and $S_{H}$ as finction of $R i$ (Figs. 1 and 2).

The critical Richardson number $R i_{c}$, beyond which stable stratification effectively suppresses the turbulence, can be found by considering the limit $e \rightarrow 0$, i.e., $G_{M} \rightarrow \infty$. In this limit, Eq.(23b) is satisfied only if the coefficient of the quadratic term vanishes, which yields

$$
R i_{c}=\frac{-c_{2}+\left(c_{2}^{2}-4 c_{1} c_{3}\right)^{\frac{1}{2}}}{2 c_{1}}
$$


Using the model constants determined in Section 6, we obtain

$$
R i_{c}=0.96
$$

Although most previous second order closure models give $R i_{c} \sim 0.2$, there is a variety of data that are in favor of a $R i_{c}$ of order one. Early laboratory data by Taylor (as cited in Monin and Yaglom, 1971) showed that turbulent exchange exists even when Ri > 1. In 1964, Webster's laboratory measurements showed that mixing persists up to $R i \sim 1$. In the oceanic PBL, Martin (1985) showed that $R i \sim 1$ is needed to obtain the correct mixed layer depth at Papa and November stations. More recently, DNS (direct numerical simulation, Gerz et al., 1989) and LES (Wang et al. 1996, Kosovic and Curry 2000) show that turibulence exists up to $R i \sim 1$. Historically, the criterion

$$
R i>\frac{1}{4}
$$

was established by Miles (1961) and Howard (1961) on the basis of linear stability analysis. However, when nonlinear interactions were included, Abarbanel et al. (1984) showed that the sufficient and necessary condition for stability is not (24c) but that

$$
R i \geq 1
$$

which is in agreement with our result (24b).

\section{Determination of model constants}

In order to determine the model constants defined in (9b), (10c) and (12c), we will employ the related time scale ratio expressions formulated in a recent theoretical turbulence model that was based in part on RNG (Renormalization Group) methods and whose predictions were tested on different flows (Canuto and Dubovikov, 1996a,b; 1997):

$$
\begin{gathered}
\lambda=\frac{\tau_{p v}}{\tau}=\frac{2}{5}, \quad \lambda_{1}=\frac{4}{15} \lambda=0.107, \quad \lambda_{5}=\frac{\tau}{\tau_{p \theta}}=5\left(1+\sigma_{t 0}^{-1}\right) \\
\lambda_{8}=\left(1-\gamma_{1}\right) \frac{\tau_{\theta}}{\tau}=\left(1-\gamma_{1}\right) \sigma_{t 0}, \quad \gamma_{1}=\frac{1}{3}
\end{gathered}
$$


where $\sigma_{t 0}$ is the turbulent Prandtl number in neutral flows and will be determined later on. To determine $\lambda_{2}, \lambda_{3}$ and $\lambda_{4}$, we adopt the following expressions (Shih and Shabbir, 1992; Canuto, 1994):

$$
\begin{aligned}
\alpha_{1}=6 \alpha_{5}, \quad \alpha_{2} & =\frac{2}{3}\left(2-7 \alpha_{5}\right), \quad \alpha_{5}=\frac{1}{10}\left(1+\frac{4}{5} F^{1 / 2}\right) \\
F & =0.64, \quad \beta_{5}=\frac{1}{2}
\end{aligned}
$$

Substituting (25a) and (25b) in (9b) yields

$$
\lambda_{2}=0.0032, \quad \lambda_{3}=0.0864, \quad \lambda_{4}=0.1
$$

We parameterize $\epsilon$ (the dissipation rate of $\epsilon$ ) as

$$
\epsilon=\frac{q^{3}}{B_{1} \ell}
$$

which corresponds to

$$
\tau=\frac{\beta_{1} \ell}{q}
$$

where the dissipation length scale $\ell \sim \kappa z$ as $z \sim 0$ and the constant $B_{1}$ is defined as $B_{1}=q^{3} / u_{*}^{3}$ where $u_{*}$ is the friction velocity, and the value of $B_{1}$ must be determined. We show that $B_{1}$ is related to the values of $\lambda_{1}, \lambda_{2}$ and $\lambda_{3}$ by assuming a logarithmic wind profile near the neutral surface layer (taking the mean wind direction as the $x$ direction). The result is:

$$
B_{1}=\left(\frac{1}{4} \lambda_{1}-\lambda_{3}^{2}+\frac{1}{3} \lambda_{2}^{2}\right)^{-3 / 4}=19.3
$$

To determine the values of $\lambda_{6}, \lambda_{7}$ and $\sigma_{t 0}$, we need some auxiliary relations. First, from $(15 \mathrm{~g}, \mathrm{i})$ an expression for the ratio of the vertical and longitudinal heat fluxes can be derived,

$$
-\frac{\overline{w \theta}}{\overline{u \theta}}=\lambda_{5} G_{M}^{-1 / 2}\left[\sigma_{t}+\frac{1}{2}\left(\lambda_{6}+\lambda_{7}\right)\right]^{-1}
$$

where $\sigma_{t} \equiv S_{M} / S_{H}$ is the turbulent Prandtl number. Webster (1964)'s experimental data show that this ratio approaches unity as $R i \sim 0$,

$$
\lambda_{5} B_{1}^{-2 / 3}\left[\sigma_{t 0}+\frac{1}{2}\left(\lambda_{6}+\lambda_{7}\right)\right]^{-1}=1
$$


where we have used the fact that $G_{M}=B_{1}^{4 / 3}$ at $R i=0$. Second, similar to the derivation of $(26 \mathrm{~b})$, in a near-neutral surface layer, from $(15 \mathrm{~g}, \mathrm{i})$ we obtain

$$
\lambda_{5}^{2}-\frac{1}{3} B_{1}^{4 / 3}\left(1+\lambda_{2}-3 \lambda_{3}\right) \sigma_{t 0} \lambda_{5}-\frac{1}{4} B_{1}^{4 / 3}\left(\lambda_{6}-\lambda_{7}\right)\left(2 \sigma_{t 0}+\lambda_{6}+\lambda_{7}\right)=0
$$

Using (10c) and (28b-c), we obtain, in the near neutral surface layer,

$$
\alpha_{3}=\frac{4}{5}+\frac{4}{5} \sigma_{t 0}\left[1-\frac{1}{3} B_{1}^{2 / 3}\left(1+\lambda_{2}-3 \lambda_{3}\right)\right]
$$

and $\lambda_{6}$ and $\lambda_{7}$ can be obtained using (28d) in (10c). We still need to determine a value for $\sigma_{t 0}$ in a consistent manner. From the third expression of (25a) and(28b-d), $\sigma_{t 0}$ is found to be related to $B_{1}, \lambda_{2}$ and $\lambda_{3}$ as follows:

$$
\sigma_{t 0}=\frac{75-3 B_{1}^{2 / 3}+3^{1 / 2}\left[1875+150 B_{1}^{2 / 3}+\left(403+400 \iota_{2}-1200 \lambda_{3}\right) B_{1}^{4 / 3}\right]^{1 / 2}}{2 B_{1}^{2 / 3}\left[3+4 B_{1}^{2 / 3}\left(1+\lambda_{2}-3 \lambda_{:}\right)\right]}=0.82
$$

So it follows that:

$$
\lambda_{5}=11.04, \quad \lambda_{6}=0.786, \quad \lambda_{7}=0.643, \quad \lambda_{8}=0.547
$$

To summarize, the model constants are determined to be:

$$
\begin{gathered}
\left(B_{1}, \lambda_{1}, \lambda_{2}, \lambda_{3}, \lambda_{4}\right)=(19.3,0.107,0.032,0.0864,0.1) \\
\left(\lambda_{5}, \lambda_{6}, \lambda_{7}, \lambda_{8}\right)=(11.04,0.786,0.643,0.547)
\end{gathered}
$$

\section{Comparison with Mellor-Yamada model and experimental data}

a. Mellor-Yamada model: a special case

The MY model (Mellor and Yamada, 1982) corresponds to:

$$
\begin{gathered}
\lambda_{1}=4\left[6\left(\frac{A_{1}}{B_{1}}\right)^{2}+B_{1}^{-4 / 3}\right], \quad \lambda_{2}=\lambda_{3}=\lambda_{4}=\frac{1}{2} \lambda=\frac{3 A_{1}}{B_{1}} \\
\lambda_{5}=\frac{B_{1}}{3 A_{2}}, \quad \lambda_{6}=\lambda_{7}=1, \quad \lambda_{8}=\frac{\tau_{\theta}}{\tau}=\frac{B_{2}}{B_{1}}
\end{gathered}
$$

Thus,

$$
\lambda=\frac{6 A_{1}}{B_{1}}, \quad \alpha_{1}=\alpha_{2}=\alpha_{3}=\gamma_{1}=0
$$


where the constants $A_{1}, B_{1}, A_{2}$ and $B_{2}$ are determined by Mellor and Yamada to be:

$$
\left(A_{1}, B_{1}, A_{2}, B_{2}\right)=(0.92,16.6,0.74,10.1)
$$

which correspond to a set of value for the model constants in the present model

$$
\begin{gathered}
\left(B 1, \lambda_{1}, \lambda_{2}, \lambda_{3}, \lambda_{4}\right)=(16.6,0.168,0.166,0.166,0.166) \\
\left(\lambda_{5}, \lambda_{6}, \lambda_{7}, \lambda_{8}\right)=(7.48,1,1,0.608)
\end{gathered}
$$

Substituting (30d) into (19), (23c) and (24a) yields

$$
R i_{c}=0.193 \quad(\text { MYmodel })
$$

One of the deficiencies of the MY model, as Mellor and Yamada pointed out themselves, is that in a neutral surface layer, the model cannot distingui:h $\overline{v^{2}}$ and $\overline{w^{2}}$, the lateral and vertical components of the velocity variance, while exper:mental data consistently show that $\overline{w^{2}}$ is always significantly smaller than $\overline{v^{2}}$. The present model solves this problem by incorporating more complete pressure correlations into the model closure so that the new model has more freedom to allow $\overline{v^{2}}$ and $\overline{w^{2}}$ to be different. To see this more closely, in a neutral surface layer, we reduce (15a-c) of the present model to:

$$
\begin{aligned}
& \frac{\overline{u^{2}}}{q^{2}}=\frac{1}{3}+\frac{\lambda_{2}+3 \lambda_{3}}{3} \\
& \frac{\overline{v^{2}}}{q^{2}}=\frac{1}{3}-\frac{2 \lambda_{2}}{3} \\
& \frac{\overline{w^{2}}}{q^{2}}=\frac{1}{3}+\frac{\lambda_{2}-3 \lambda_{3}}{3}
\end{aligned}
$$

In the MY model $\lambda_{2}=\lambda_{3}$, which makes $\overline{v^{2}}=\overline{w^{2}}$, while in the present model $\lambda_{2}$ and $\lambda_{3}$ are two independent parameters, and we choose to determine them according to Shih and Shabbir (1992)'s expressions that are derived from theoretical considerations and have been shown to be consistent with measured data.

\section{b. Comparison with measured and LES data}

The turbulent Prandtl number, $\sigma_{t}=K_{M} / K_{H}^{\prime}$, is one of the important parameters of turbulence. We compare the inverse of $\sigma_{t}$ as a function of the gradient Richardson 
number $R i$ resulting from both the present model and the MY model with the experimental data of Webster (1964). It is clear that turbulence in the stably stratified flow exists well beyond the MY critical value $R i \approx 0.2$. According to the experimental data, the critical value of $R i$ should be of order unity, and the present model falls within the range of the measured data (Fig.3).

We also compare the vertical and lateral heat flux ratio $-\overline{w \theta} / \overline{u \theta}$ (as a function of $R i$ ) resulting from both the present model and the MY model with the experimental data of Webster (1964). Webster described the ratio as "(being) seen to fall catastrophically from unity in neutral conditions to only about 0.5 at $R i$ equal to 0.2 and even less for higher Richardson numbers." The present model gives the critical Richardson number $R i_{c}=0.96$, in agreement with the data (Fig.4).

We then compare the present model with the recent LES of Kosovic and Curry (2000) on a stably stratified PBL, at hour 12 of the high-resolution case NLHRB, when a quasi-steady state is reached. In our simulation we use the level 2 model since we are particularly interested in the behavior of the model when the grartient Richardson number $R i$ varies; for the length scale formula we use

$$
\begin{aligned}
& \ell_{0}=0.1 \frac{\int_{0}^{\infty} z q d z}{\int_{0}^{\infty} q d z}, \quad \ell_{1}=\frac{\kappa z \ell_{0}}{\ell_{0}+\kappa z} \\
& \ell=\left\{\begin{array}{cc}
\ell_{1} & : \quad \frac{\partial \Theta}{\partial z} \leq 0 \\
\min \left(\ell_{1}, 0.53 \frac{q}{N}\right) & : \frac{\partial \Theta}{\partial z}>0
\end{array}\right.
\end{aligned}
$$

One can see from figures 5-7 that the present model is more consistent with the LES result than the MY model, as we will discuss below.

It is very informative to examine the nondimensional shear and potential temperature gradients defined as

$$
\Phi_{m}=\frac{\kappa z}{u_{*}} S, \quad \Phi_{h}=\frac{\kappa z u_{*}}{\left|\overline{w \theta}_{s}\right|} \frac{\partial \Theta}{\partial z}
$$

where $u_{*}$ and $\overline{w \theta}_{s}$ are the friction velocity and the surface potential temperature flux respectively and $S$ is the shear given by Eq.(18b). In Figs. 5 and 6 we plot $1 / \Phi_{m}$ and $1 / \Phi_{h}$ as function of $R i$. The graphs indicate that the present model can reproduce the observed Kansas data as analyzed by Businger et al. 1971) in the context of 
Monin-Obukhov similarity theory for $R i<0.2$. For $R i>0.2$, the present model shows that turbulence still exists although is weaker, in agreement with Kosovic and Curry (2000)'s LES data. This result is also in agreement with Brown et al. (1994) and Andren (1995). In the same figures we also plot the results of the MY model, which deviate from the Kansas data for $R i<0.2$, and fail to reproduce the turbulence beyond $R i=0.2$ found in the LES.

The PBL height is one of the most important quantities in any PBL modeling. The PBL height is usually defined as the height at which the turbulent kinetic energy or the magnitude of the momentum flux decreases to a small fraction of the corresponding surface value; or it may be defined as the height at which the (positive) temperature gradient reacheas a certain value from below. In any case, the top of the PBL lies in a region where the turbulence is stably stratified and, given the mean profiles of the wind and the temperature (and thus given $R i$ ), a higher intensity level of turbulence yields a greater PBL height. The MY model, however, underestimates the PBL height (Yamada and Mellor 1975). Since the present model predicts larger critical Richardson number and produces more turbulence for a given Richardson number, greater PBL heights can be achieved (Fig.7).

\section{Conclusions}

With a single new input, the most updated expressions for the pressure-velocity and pressure-temperature correlations, we have derived a second-order closure turbulence model to describe the PBL. One of the main features of the new model is that it yields a critical Richardson number of order unity, rather than $\sim 0.2$ as given by most previous models. The larger critical Richardson number is in agreement with measured and LES data and the stability analysis that includes non-linear interactions. The new model reproduces the Kansas data as analyzed by Businger et al. (1971) for Richardson numbers smaller than 0.2 as well as the LES and laboratory data for Richardson numbers up to unity. Another improvement is that the present model allows a match of the surface data, which was not possible in previous second order closure PBL models. In addition, the new model produces greater PBL height than the previous models. 


\section{Figure Captions}

Fig.1. The stability function $S_{M}$ versus the gradient Richardson number $R i$. The solid line represents the present model; the dotted line, the MY model.

Fig.2. The stability function $S_{H}$ versus the gradient Richardson number $R i$. The solid line represents the present model; the dotted line, the MY model.

Fig.3. The inverse turbulent Prandtl number $\sigma_{t}^{-1}$ (normalized by its value for neutral stratification) versus the gradient Richardson number. The solid line is the result of the present model at level 2. The dotted line represents the level $2 \mathrm{MY}$ model. The experimental data by Webster (1964) are redrawn here as filled circles. The present model yields a much larger critical Richardson number $(\approx 1)$ than the Mellor-Ýamada model $(\approx 0.2)$.

Fig.4. Ratio of the rates of heat transport in the w-direction (vertical) and the $u$-direction (horizontal, along the mean flow), $-\overline{w \theta} / \overline{u \theta}$, versus the Richardson number. The solid line represents the result of the present model, while the dotted line represents the MY model. The experimental data (Webster, 1964) are redrawn here as filled circles.

Fig.5. Nondimensional shear as a function of the gradient Richardson number. The crosses represent the LES simulation of Kosovic and Curry (2000), case NLHRB (their Eq. 2i). The solid line represents simulation results using the present model, while the dotted line, simulation results using the MY model. The dashed line represents the measured Kansas data (Businger et al. 1971).

Fig.6. Similar to Fig.5 but for the nondimensional potential temperature gradient (Eq. 28 of Kosovic and Curry, 2000).

Fig.7. PBL height as a function of the dimensionless time $t f_{c}$, where $f_{c}$ is the

Coriolis parameter. Cross: LES result; solid line: present model result; dotted line: 
MY model result.

\section{Appendix}

\section{The Level 3 PBL model}

In the level $3 \mathrm{PBL}$ model, the turbulent temperature variance $\overline{\theta^{2}}$ is solved from its prognostic equation (instead of from an algebraic equation):

$$
\frac{D}{D t} \overline{\theta^{2}}+\frac{\partial}{\partial z} \overline{w \theta^{2}}=-2 \frac{\partial \Theta}{\partial z} \overline{w \theta}-2 \frac{\overline{\theta^{2}}}{\tau_{\theta}}
$$

From (10a), the algebraic equation for the heat flux $\overline{w \theta}$ is:

$$
\overline{w \theta}=-\lambda_{5}^{-1} \tau\left[\frac{\partial \Theta}{\partial z} \overline{w^{2}}+\frac{1}{2}\left(\lambda_{6}-\lambda_{7}\right)\left(\frac{\partial U}{\partial z} \overline{u \theta}+\frac{\partial V}{\partial z} \overline{v \theta}\right)\right]+\lambda_{5}^{-1} \lambda_{0} g \alpha \tau \overline{\theta^{2}}
$$

All the other algebraic equations for the Reynolds stress and the heat flux are the same as the level 2.5 model, $(15 \mathrm{a}-\mathrm{h})$, except (15i), which is replaced by (A2) in the level 3 model. We solve (15a-h) and (A2) using symbolic algebra and the results are:

$$
(\overline{u w}, \overline{v w})=-\epsilon \tau S_{M}\left(\frac{\partial U}{\partial z}, \frac{\partial V}{\partial z}\right), \quad \overline{w \theta}=-\epsilon \tau S_{H} \frac{\partial \Theta}{\partial z}+\gamma_{c}
$$

where

$$
\gamma_{c}=\frac{3\left[\lambda_{5}+\lambda_{4} G_{H}+\lambda_{5}\left(\lambda_{3}^{2}-\frac{1}{3} \lambda_{2}^{2}\right) G_{M}\right]}{D} \lambda_{0} g \alpha \tau \overline{\theta^{2}}
$$

is the counter-gradient term which is absent in the level 2.5 model and $D$ is the same as in (18c). The structure of the stability function $S_{M}$ differs from the $S_{M}$ in the level 2.5 model (17a) by an extra term,

$$
S_{M}=\frac{1}{D}\left[s_{0}+s_{1} G_{H}+s_{2} G_{M}+s_{3} \lambda_{0}(g \alpha \tau)^{2} \frac{\overline{\theta^{2}}}{e}\right]
$$

where

$$
s_{3}=\frac{1}{2} \lambda_{4}\left[2 \lambda_{5}\left(\lambda_{2}+3 \lambda_{3}\right)+3\left(\lambda_{6}+\lambda_{7}\right)\right]
$$

The form of the stability function $S_{H}$ remains the same as that of the level 2.5 model (17b). The expressions for the model constants are the same as in (19) except that in level 3 model $\lambda_{8}=0$. In addition, the constant $\lambda_{0}$ needed in (A4) is set to $2 / 3$ according to $(10 \mathrm{c})$ and $(25 \mathrm{a})$. 


\section{Acknowledgements}

The authors thank Drs. B. Kosovic and J.A. Curry for providing their LES data to be used in this paper.

\section{References}

Abarbanel,H.D., D.D. Holm, J.E. Marsden and T. Ratiu, 1984: Richardson number criterion for the non-linear stability of 3D stratified flow, Phys. Res. Lett., 52, 23522355.

Andren, A., 1995: The structure of stably stratified atmospheric boundary layers: A large-eddy simulation study. Quart. J. Roy. Meteor. Soc., 121, 961-985.

Brown, A.R., S.H. Derbyshire and P.J. Mason 1994: Large-eddy simulation of stable atmospheric boundary layers with a revised stochastic subgrid model. Quart. J. Roy. Meteor. Soc., 120, 1485-1512.

Businger, J.A., J.C. Wyngaard, Y. Izumi and E.F. Bradley 1971: Flux-profile relationships in the atmospheric surface layer. J. Atmos. Sci., 28, 181-189.

Canuto,V.M., 1994: Large eddy simulation of turbulence: a subgrid model including shear, vorticity, rotation and buoyancy. Ap. J., 428, 729-752.

Canuto,V.M., Y. Cheng and A.M. Howard, 2000: New third-order moments for convective PBL. J. Atmos. Sci. (in press).

Canuto,V.M., and M.S. Dubovikov, 1996a: A dynamical model for turbulence. I general formalism. Physics of Fluids, 8, 571-586.

Canuto,V.M., and M.S. Dubovikov, 1996b: A dynamical model for turbulence. II shear-driven flows. Physics of Fluids, 8, 587-598.

Canuto,V.M., and M.S. Dubovikov, 1997: A dynamical model for turbulence. IV 
buoyancy driven flows. Physics of Fluids, 9, 2118-2131.

Canuto, V.M., F. Minotti, C. Ronchi, R.M. Ypma and O. Zeman, 1994: Second-order closure PBL model with new third-order moments: comparison with LES data. $J$. Atmos. Sci., 51, 1605-1618.

Cheng, Y., and V.M. Canuto, 1994: Stably stratified shear turbulence: a new model for the energy dissipation length scale. J. Atmos. Sci., 51, 2384-2396.

Chou, P.Y., 1940: On an extension of Reynolds' method of finding apparent stress and the nature of turbulence. Chinese Journal of Physics, 4(1), 1-33.

Chou, P.Y., 1945: On velocity correlations and the solutions of the equations of turbulent fluctuation. Quarterly of Applied Mathematics, 3(1), 38-54.

Gerz, T., U. Schumann and S.E. Elghobashi, 1989: Direct numerical simulation of stratified, homogeneous turbulent shear flows. J. Fluid Mech., 220, 563-594.

Hassid. S.. and B. Galperin, 1983: A turbulent energy model for geophysical flows. Boundary-Layer Meteorol, 26, 397-412.

Howard, L.N., 1961: Note on a paper by J.W. Miles. J. Fluid Mech., 10, 509-512.

Hunt, J.C.R., D.D. Stretch and R.E. Britter, 1988: Length scales in stably stratified turbulent flows and their use in turbulence models. In Stably Stratified Flows and Dense Gas Dynamics, Ed. J. S. Puttock, Clarendon Press, 285-321.

Kosovic, B., and J.A. Curry, 2000: A large eddy simulation of a quasi-steady, stably stratified atmospheric boundary layer. J. Atmos. Sci, 57, 1052-1068.

Launder, B. E., G. Reece and W. Rodi, 1975: Progress in the development of a Reynolds-stress turbulent closure. J. Fluid Mech., 68, 537-566.

Lumley, J. L., 1978: Computational modeling of turbulent flows. Adv. in Applied 
Mech., 18, 123-176.

Lumley, J.L., and B. Khajeh-Nouri, 1974: Computational modeling of turbulent transport. Advances in Geophysics, 18A, Academic Press, 169-192.

Martin, P.J., 1985: Simulation of the mixed layer at OWS November and Papa with several models. J. Geophy. Res., 90, 903-916.

Mellor, G.L., 1973: Analytic prediction of the properties of stratified planetary surface layers. J.Atmos.Sci., 30, 1061-1069.

Melior, G.L., and T. Yamada, 1974: A hierarchy of turbulence closure models for planetary boundary layer. J. Atmos. Sci., 31, 1791-1806.

Melior, G.L., and T. Yamada, 1982: Development of a turbulence closure model for geophysical fluid problems, Rev. Geophys. Space Phys., 20, 851-875.

Mile;, J.W., 1961: On the stability of heterogeneous shear flows. J. Fluid Mech., 10, 496-508.

Monin, A.S. and A.M. Yaglom, 1971: Statistical fluid mechanics. Vol. 1, 2, MIT Press.

Nieuw'stadt, F.T.M., 1984: The turbulent structure of the stable, nocturnal boundary layer. . J. Atmos. Sci., 41, 2202-2216.

Nieuwstadt, F.T.M., 1985: A model for the stationary, stable boundary layer. Turbulence and Diffusion in Stable Environments, J.C.R. Hunt, Ed., Clarendon Press, 149-179.

Pope, S. B., 1975: A more general effective viscosity hypothesis. J. Fluid Mech., 72, 331.

Rotta, J.C., 1951:Statistische theorie nichthomogener turbulenz. Z. Phys., 129,547. 
Shih,T-H., and A. Shabbir, 1992: Advances in modeling the pressure correlation terms in the second moment equations. In Studies in Turbulence, Ed. T.B. Gatski, S. Sarkar and C.G. Speziale, 91-128.

Speziale, C. G., 1991: Analytical methods for the development of Reynolds-stress closures in turbulence. Ann. Rev. Fluid. Mech., 23, 107-157.

Wang, D., W.G. Large and J.C. McWilliams, 1996: Large-eddy simulation of the equatorial ocean boundary layer: diurnal cycling, eddy viscosity, and horizontal rotation. J. Geophy. Res., 101, 3649-3662.

Webster, C.A.G., 1964: An experimental study of turbulence in a density stratified shear flow. J. Fluid Mech., 19, 221-245.

Zeman, O., and J.L. Lumley, 1979. Buoyancy effects in entraining turbulent boundary layers: a second-order closure study. In Turbulent Shear Flows I, Ed. F. Durst, B.E.Launder, F.W.Schmidt, J.H.Whitelaw, Berlin:Springer-Verlag, 295-302. 


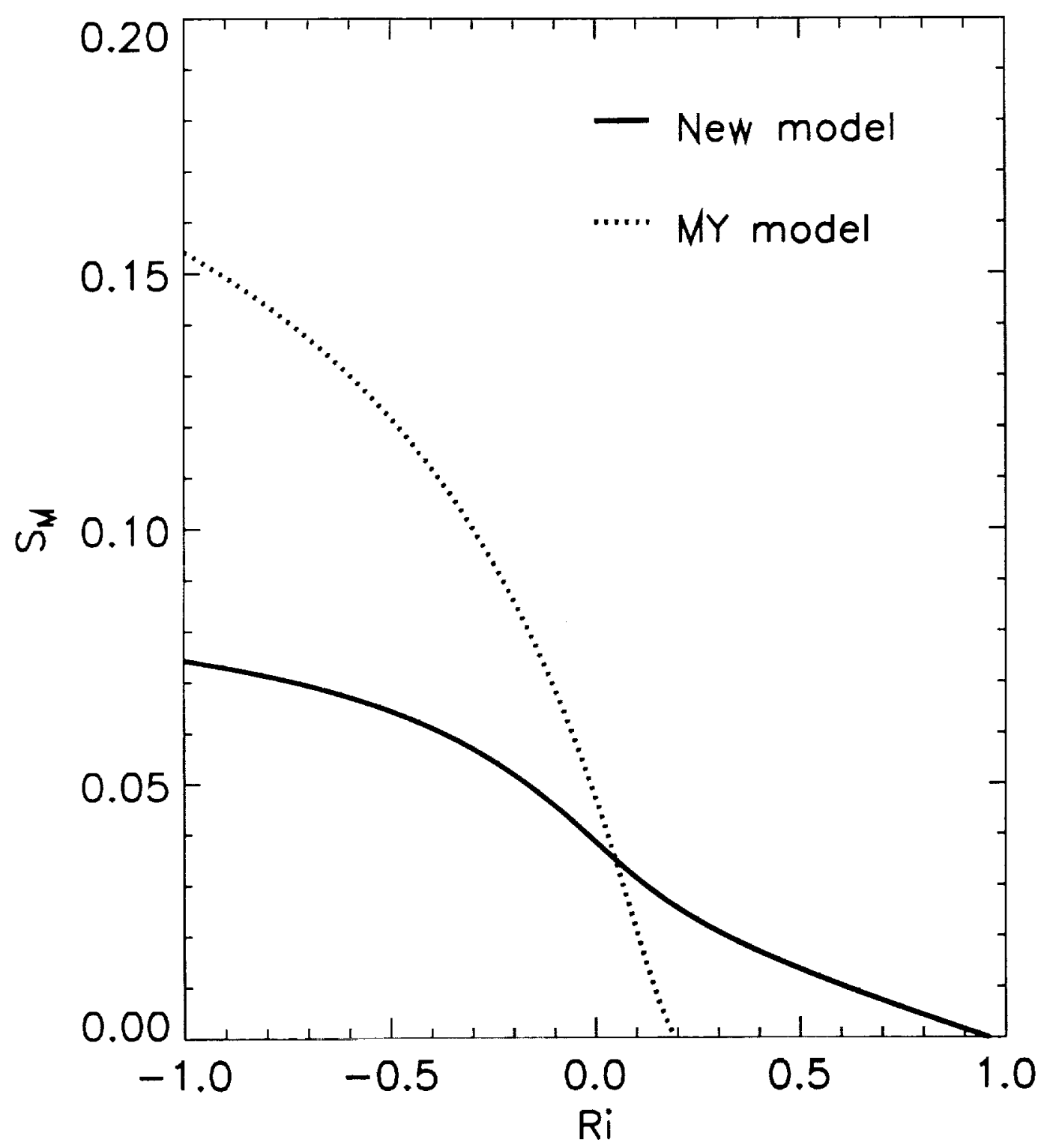

Fig. 1 


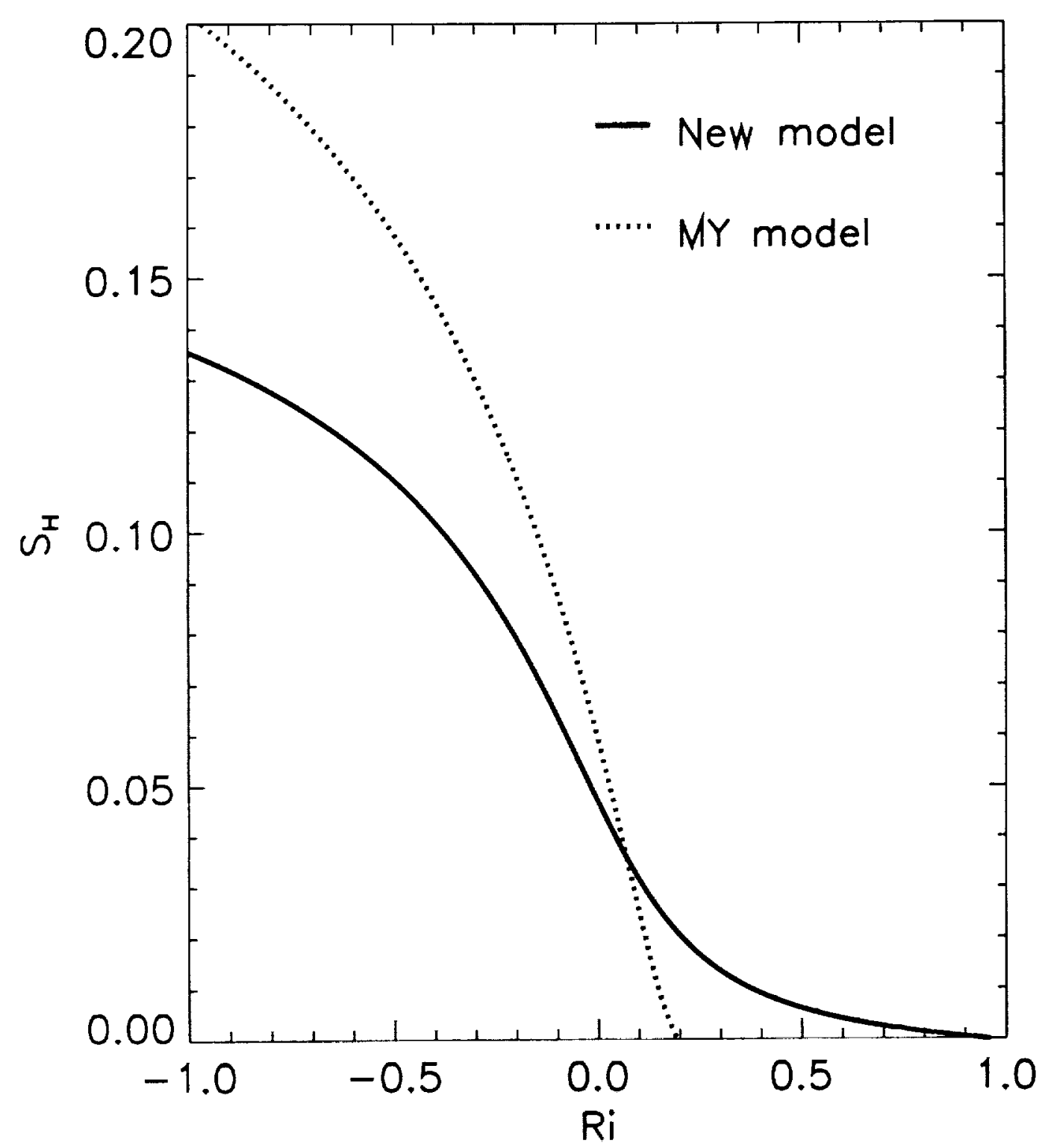

Fig. 2 


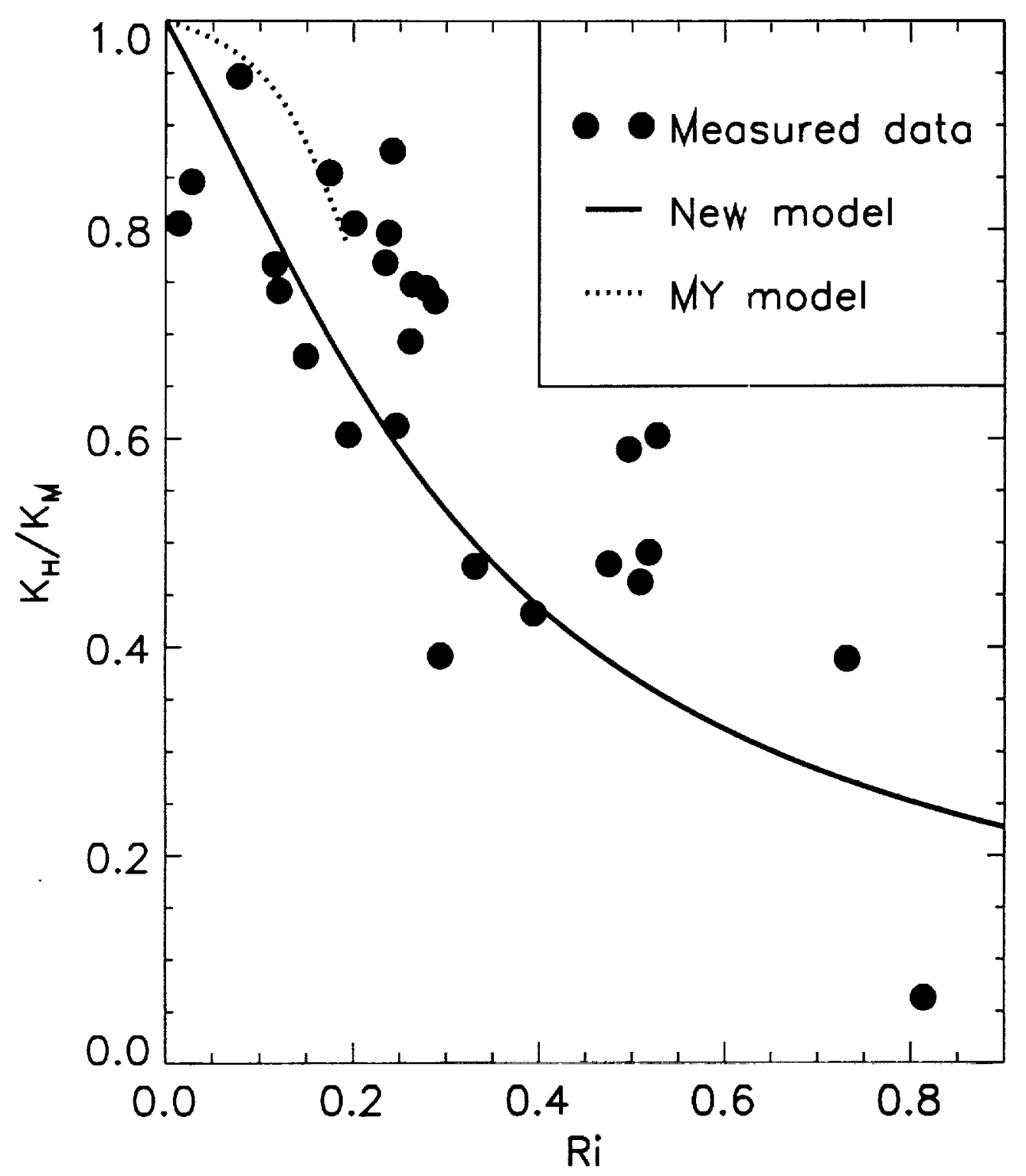

Fig. 3 


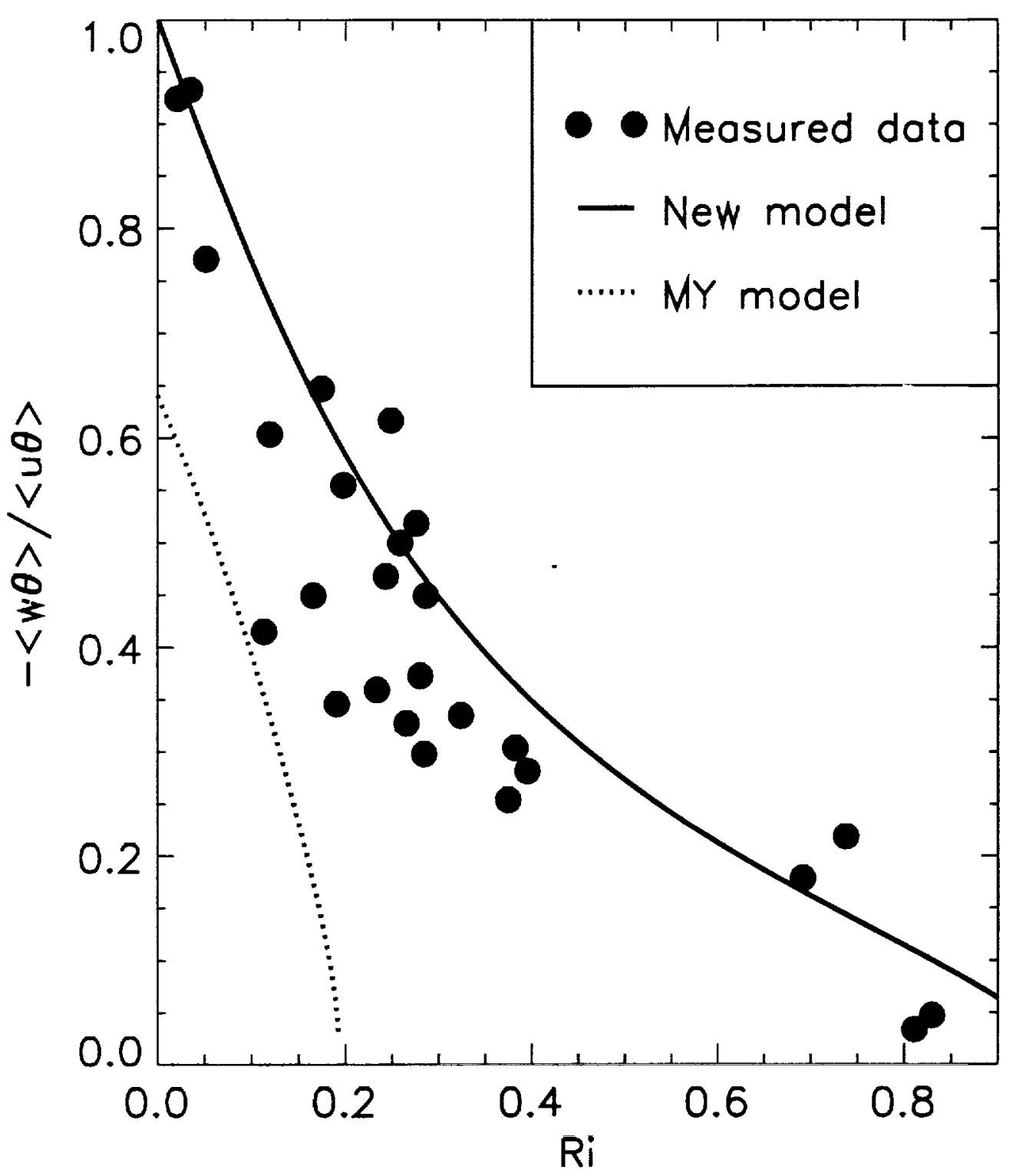

Fig. 4 


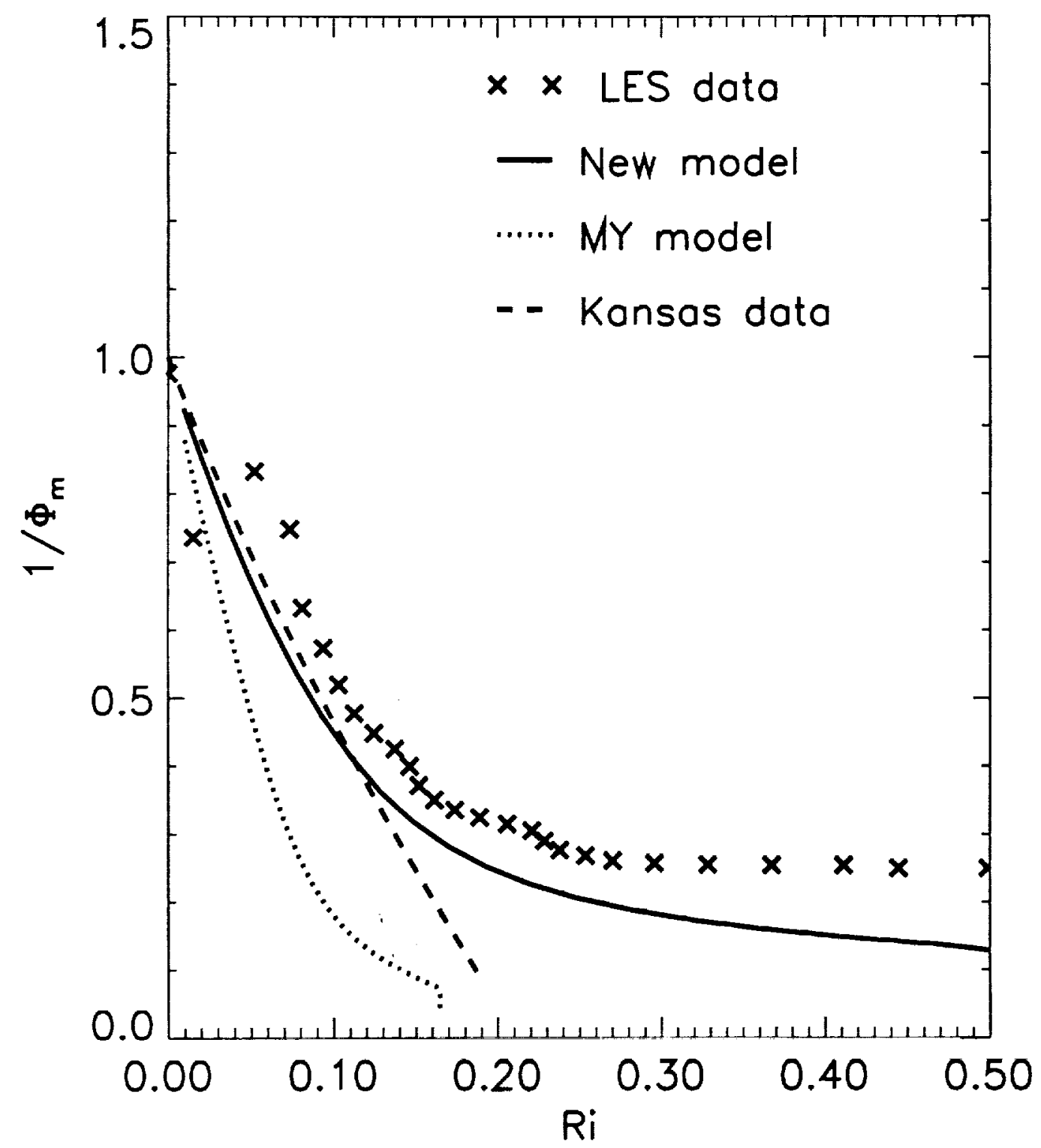

Fig. 5 


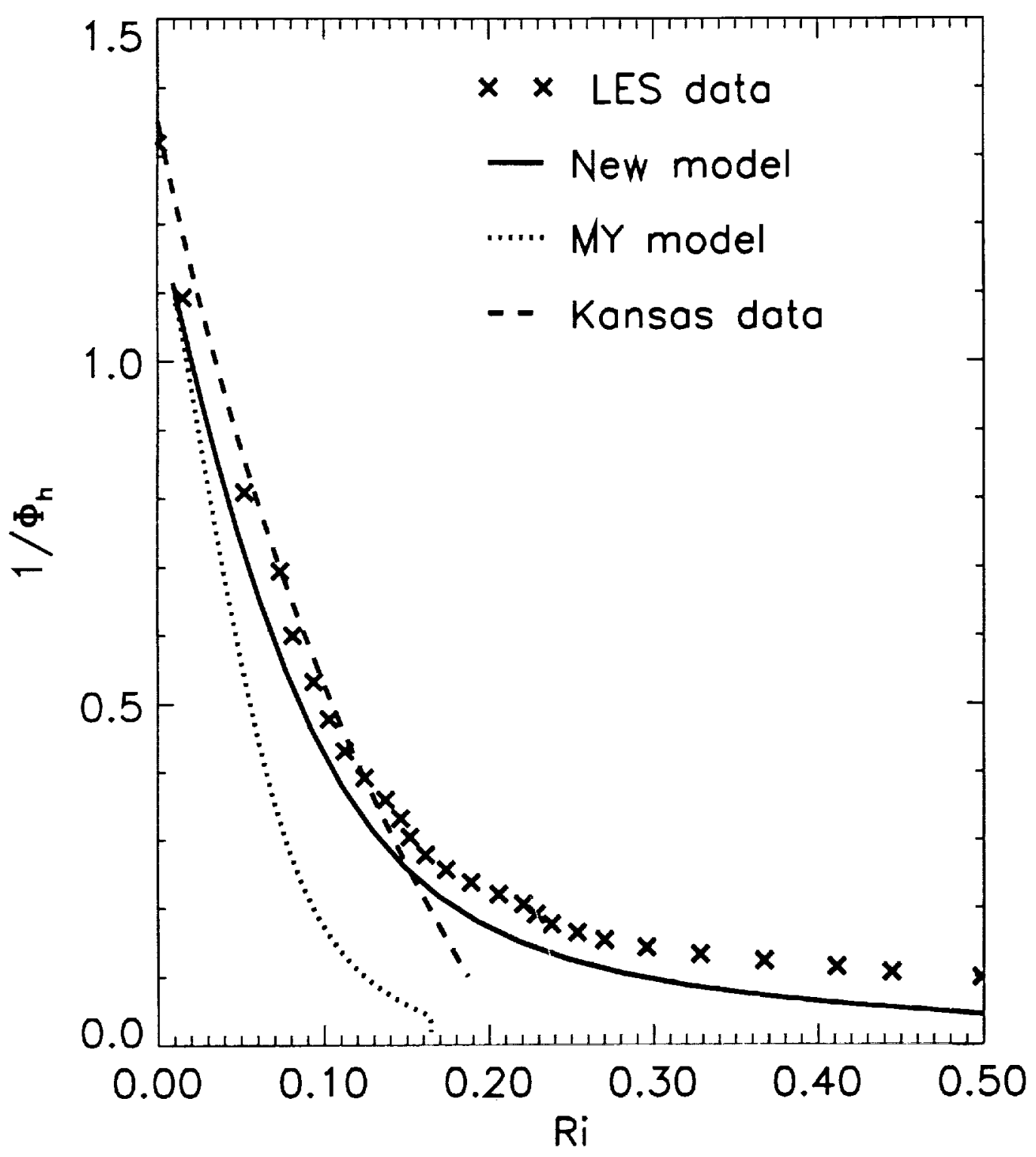

Fig. 6 


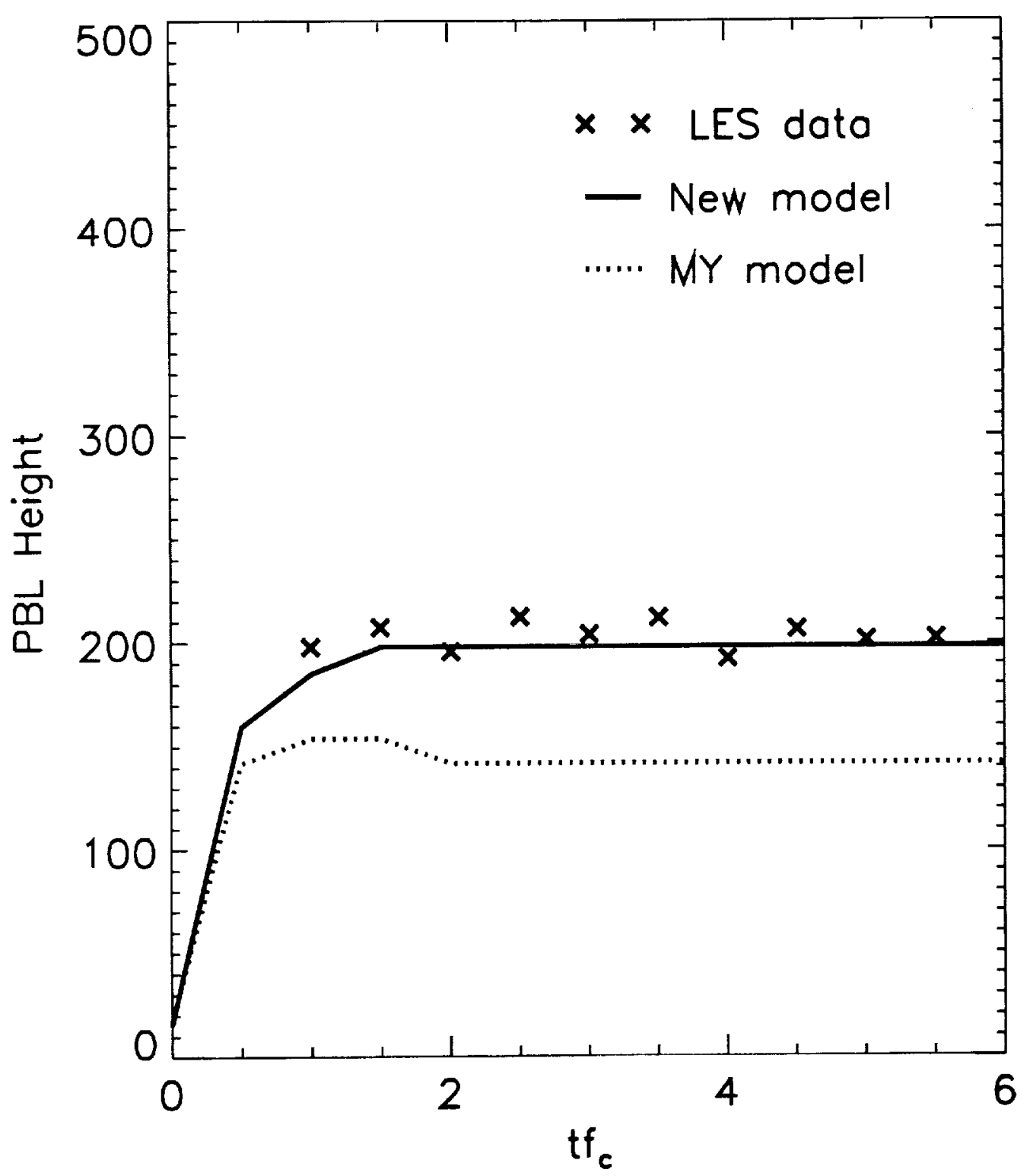

Fig. 7 